\title{
Prediction of large bias-dependent magnetoresistance in all-oxide magnetic tunnel junctions with a ferroelectric barrier
}

\author{
Nuala M. Caffrey, Thomas Archer, Ivan Rungger, and Stefano Sanvito* \\ School of Physics and CRANN, Trinity College, Dublin 2, Ireland
}

(Received 10 December 2010; published 17 March 2011)

\begin{abstract}
All-oxide magnetic tunnel junctions (MTJs) incorporating functional materials as insulating barriers have the potential of becoming the founding technology for novel multifunctional devices. We investigate, by first-principles density functional theory, the bias-dependent transport properties of an all-oxide $\mathrm{SrRuO}_{3} / \mathrm{BaTiO}_{3} / \mathrm{SrRuO}_{3} \mathrm{MTJ}$. This incorporates a $\mathrm{BaTiO}_{3}$ barrier which can be found either in a nonferroic or in a ferroelectric state. In such an MTJ not only can the tunneling magnetoresistance reach enormous values, but also, for certain voltages, its sign can be changed by altering the barrier electric state. These findings pave the way for a new generation of electrically controlled magnetic sensors.
\end{abstract}

DOI: 10.1103/PhysRevB.83.125409

\section{INTRODUCTION}

The control of the spin-dependent tunneling between two ferromagnetic electrodes separated by an insulating barrier has enabled enormous advances in many magnetic data storage technologies, in particular since extremely large tunneling magnetoresistance (TMR) was measured. The progress in producing magnetic tunnel junctions (MTJs) with large TMR was initially limited by the use of amorphous tunnel barriers. The situation, however, changed after the prediction ${ }^{1,2}$ and subsequent experimental realization ${ }^{3,4}$ of epitaxial MTJs. Since then, room temperature TMR in excess of $600 \%$ has been demonstrated in $\mathrm{MgO}$-based devices. ${ }^{5}$

In general, for amorphous barriers the spin polarization of the tunneling current and hence the TMR magnitude, depend solely on the electrodes' density of states (DOS) at the Fermi level, $E_{\mathrm{F}}{ }^{6}{ }^{6}$ In contrast, perfectly crystalline tunnel barriers are wave-function symmetry selective and make the tunneling process sensitive to their electronic structure. As a result the amplitude and even sign of the TMR may depend on the barrier itself. The understanding of such a concept suggests that one can engineer the TMR by carefully selecting the insulating barriers to be epitaxially grown on magnetic electrodes. Ferromagnets ${ }^{7}$ and ferroelectrics ${ }^{8,9}$ are of particular interest as functional barriers.

Ferroelectric materials possess a spontaneous electric polarization whose direction can be switched by an electric field. This makes ferroelectric-based MTJs fully multifunctional devices able to respond to both electrical and magnetic stimuli. ${ }^{9,10}$ Importantly, ferroelectrics can be grown epitaxially on a variety of substrates, ${ }^{11}$ but in particular on other oxides. Since epitaxial growth is a prerequisite for large TMR, the prospect of all-oxide junctions appears particularly attractive. Such a type of MTJ is investigated in this article. We demonstrate theoretically a huge TMR and more importantly we show that the TMR sign can be reversed with bias, at a critical bias which depends on the ferroic state of the barrier. Our results are rationalized in terms of the band-structure match between the ferroelectric insulator and the ferromagnetic electrodes.

\section{COMPUTATIONAL DETAILS}

Density functional theory (DFT) calculations are performed with the local basis set code SIESTA. ${ }^{12}$ Structural relaxation is obtained with the generalized gradient approximation (GGA) of the exchange and correlation functional. ${ }^{13}$ This gives a satisfactory device geometry, but it produces a rather shallow band alignment mainly because of the DFT-GGA gap problem. This is problematic when applying a bias across the junction as the conduction band will quickly become populated leading to an underestimation of the breakdown electric field. ${ }^{14}$ To make up for this shortfall the electronic structure used for the transport calculations is that obtained with the atomic self-interaction correction (ASIC) scheme, ${ }^{15}$ which improves drastically the electronic properties of both bulk $\mathrm{BaTiO}_{3}$ (Ref. 16) and $\mathrm{SrRuO}_{3}$ (Ref. 17) while increasing the electric field at which the device will breakdown. Unfortunately the approximate ASIC energy functional is not sufficient to produce good structural parameters and, in particular, the $\mathrm{BaTiO}_{3}$ ferroelectric state cannot be stabilized. This is a current limitation of the method, which otherwise has been successful in predicting the electronic properties of oxides. ${ }^{18}$ For this reason we perform ASIC transport calculations at the GGA relaxed structural parameters to provide the most realistic junction description. For all the calculations we use a $6 \times 6 \times 1$ $k$-point Monkhorst-Pack mesh to converge the density matrix to a tolerance of $1 \times 10^{-5}$ and a grid spacing equivalent to a plane-wave cutoff of $800 \mathrm{eV}$.

Electron transport is computed with the SMEAGOL code, ${ }^{19,20}$ which combines the nonequilibrium Green's function scheme with DFT. Since SMEAGOL interfaces SIESTA as the DFT platform, we employ here the same parameters used for the total energies calculations. In brief, the total electronic current is given by

$$
I^{\sigma}(V)=\frac{e}{h} \int d E T^{\sigma}(E ; V)\left[f_{L}-f_{R}\right],
$$

where $\sigma$ labels the spin $(\uparrow, \downarrow), T^{\sigma}(E ; V)$ is the energydependent transmission coefficient for the bias $V, f_{L / R}$ is the Fermi distribution function evaluated at $E-\mu_{L / R}$, and $\mu_{L / R}=E_{F} \pm \frac{e V}{2}$ is the chemical potential of the left/right electrode. If the junction is perfectly translational invariant in the plane orthogonal to the transport direction, $T^{\sigma}$ is obtained by integrating the $\vec{k}$ dependent $T_{\vec{k}}^{\sigma}$ over the two-dimensional (2D) Brillouin zone of volume $\Omega_{\mathrm{BZ}}$,

$$
T^{\sigma}(E, V)=\frac{1}{\Omega_{\mathrm{BZ}}} \int_{\mathrm{BZ}} d \vec{k} T_{\vec{k}}^{\sigma}(E ; V) .
$$


$T^{\sigma}(E, V)$ is evaluated on a $100 \times 100 \times 1 k$-point MonkhorstPack mesh for the zero-bias calculations and on a $24 \times 24 \times 1$ $k$-point mesh for the bias calculations. No sensible changes in $T^{\sigma}(E, V)$ were found when enlarging these $k$-point meshes.

\section{STRUCTURE}

We perform an initial relaxation of bulk $\mathrm{BaTiO}_{3}$ and $\mathrm{SrRuO}_{3}$ under an in-plane compressive strain, emulating the common epitaxial growth on $\mathrm{SrTiO}_{3}$, to find the relaxed out-of-plane lattice constant. The relaxed cells are then used to construct the transport supercell, which comprises six $\mathrm{BaTiO}_{3}$ unit cells $(\sim 2.5 \mathrm{~nm})$ sandwiched at either side by three $\mathrm{SrRuO}_{3}$ ones. The $\mathrm{SrRuO}_{3} / \mathrm{BaTiO}_{3}$ interface is $\mathrm{SrO} / \mathrm{TiO}_{2}$ due to the experimentally observed volatility of the $\mathrm{RuO}_{2}$ termination. $^{21,22}$ We consider two structures. In the first nonferroic (NFE) structure the atoms are frozen artificially in their centrosymmetric positions with the interfacial distance given by an average between the $\mathrm{BaTiO}_{3}$ and $\mathrm{SrRuO}_{3} c$-lattice constant. In the second all positions in the supercell are completely relaxed out-of-plane until the forces are less than $10 \mathrm{meV} / \AA$, resulting in a stable ferroelectric ground state (FE structure).

The atomic relaxed displacements $\delta$ with respect to the planar $\mathrm{O}$ positions are shown in Fig. 1. At the center of the the $\mathrm{BaTiO}_{3}$ slab Ti displaces by $0.14 \AA$, which is significantly smaller than the value of $0.23 \AA$ of bulk $\mathrm{BaTiO}_{3}$ experiencing the same strain. Note that GGA overestimates the volume and atomic distortions associated with ferroelectricity in $\mathrm{BaTiO}_{3}$ resulting in a "supertetragonal" structure. Such an overestimation, while resulting in a polarization greater than the experimental one, will not have a significant qualitative effect on our results. The interfacial $\mathrm{SrRuO}_{3}$ layers, as expected, also contribute to the polarization. ${ }^{23}$

\section{RESULTS AND DISCUSSIONS}

\section{A. Band structure and symmetries}

The symmetry of the electronic bands of both the ferromagnetic electrodes and the insulating spacer dictates the transport properties. A wave function, whether propagating or evanescent, is described in terms of irreducible representations of the crystal's symmetry group. For a cubic space group, the $\Delta_{1}$ symmetry transforms as a linear combination of 1 ,

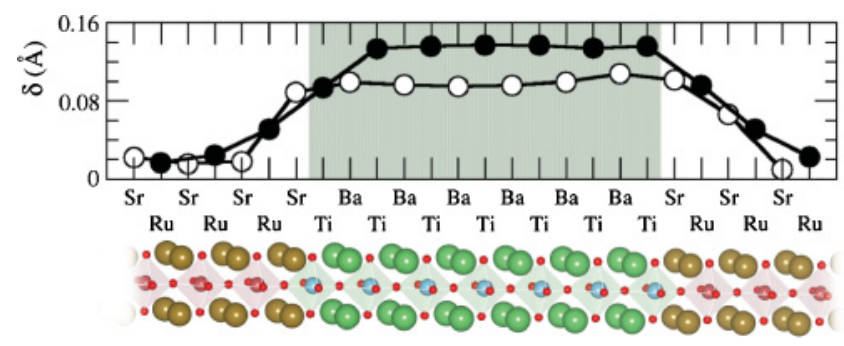

FIG. 1. (Color online) Relative atomic displacement for $\mathrm{Sr}$, $\mathrm{Ba}$ (open circle) and $\mathrm{Ru}, \mathrm{Ti}$ (filled circle) in the relaxed $\mathrm{SrRuO}_{3} / \mathrm{BaTiO}_{3} / \mathrm{SrRuO}_{3} \mathrm{MTJ}$ investigated. The displacements are with respect of the $\mathrm{O}$ atoms in the same plane, $\delta=\left(z_{\text {cation }}-z_{\mathrm{O}}\right)$, with $z$ being the stack direction. The device geometry is presented in the lower part of the figure.

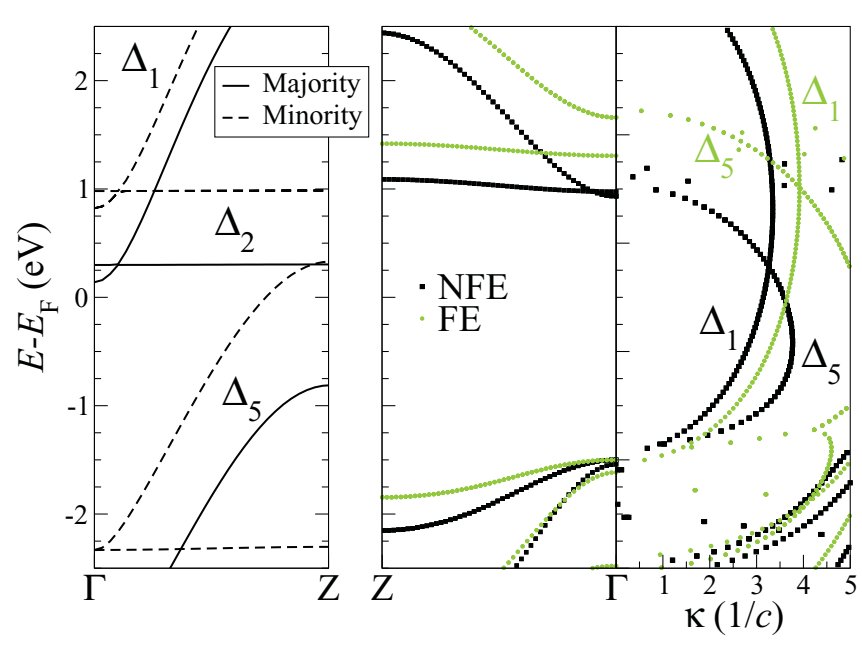

FIG. 2. (Color online) ASIC-calculated band structure along the transport direction $(\Gamma \rightarrow Z)$ for centrosymmetric tetragonal $\mathrm{SrRuO}_{3}$ (left), and $\mathrm{BaTiO}_{3}$ (middle) both in the NFE (black) and FE (green) configuration. The wave-function symmetries of the bands close to $E_{\mathrm{F}}$ are indicated. The right panel reports the complex band structure for $\mathrm{BaTiO}_{3}$. The energies are aligned with the $E_{\mathrm{F}}$ of $\mathrm{SrRuO}_{3}$.

$z$ and $2 z^{2}-x^{2}-y^{2}$ functions, while the $\Delta_{5}$ as a linear combination of $z x$ and $z y$ (e.g., $p_{x}, p_{y}, d_{x z}$ and $d_{y z}$ ). Finally, the $d_{x^{2}-y^{2}}$ and $d_{x y}$ states have $\Delta_{2}$ and $\Delta_{2^{\prime}}$ symmetry, respectively. Importantly, an incident Bloch state in the electrodes can couple to a given evanescent state in the insulator and then sustain a tunneling current, only if the two share the same symmetry.

The left panel of Fig. 2 shows the $\mathrm{SrRuO}_{3}$ band structure close to $E_{\mathrm{F}}$ along the direction of the transport. At $E_{\mathrm{F}}$ only a doubly degenerate minority $\Delta_{5}$ state is available, in contrast to previous DFT calculations, where both minority $(\downarrow) \Delta_{5}$ and majority $(\uparrow) \Delta_{1}$ bands were found. ${ }^{10}$ Such a discrepancy is due to the use of the GGA functional in Ref. 10, which underestimates the $\mathrm{Ru} d$ manifold exchange splitting. ${ }^{17}$ Note that a large spin splitting is expected based on point contact Andreev reflection experiments. ${ }^{24}$

In the right panel of Fig. 2 we plot the $\mathrm{BaTiO}_{3}$ real and complex band structure. In contrast to $\mathrm{MgO}$, where states with $\Delta_{5}$ symmetry decay significantly faster than those with $\Delta_{1}$ (Ref. 1), in $\mathrm{NFE} \mathrm{BaTiO}_{3}$ the $\Delta_{1}$ and $\Delta_{5}$ symmetries have comparable decay rates. In particular, close to the valence band top the slower decay rate is for $\Delta_{1}$, while the situation is reversed at the conduction band minimum. The enlargement of the band gap associated with the FE order results in an increased decay rate for all the symmetries. The effect is more pronounced for $\Delta_{5}$ close to the top of the valence band where now the $\Delta_{1}$ symmetry primarily contributes to the tunnel conductance.

\section{B. Zero-bias transport properties}

We begin our analysis of the transport properties from the NFE structure by showing $T(E)$ at zero bias for the parallel (PA) and antiparallel (AP) magnetic alignment of the electrodes (Fig. 3). In the PA configuration $T(E)$ close to $E_{\mathrm{F}}$ is dominated by the minority spin channel. This is expected from the band structure of $\mathrm{SrRuO}_{3}$, which presents only a 


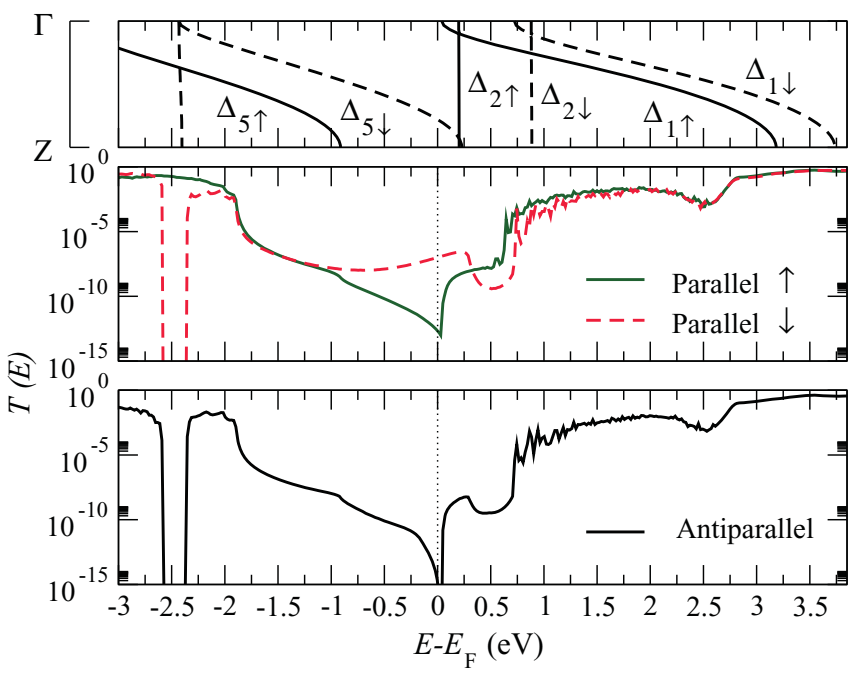

FIG. 3. (Color online) Transmission coefficients $T(E)$ as a function of energy for the NFE structure. The middle panel is for the parallel magnetic configuration, while the lower one is for the antiparallel. At the top we report again the $\mathrm{SrRuO}_{3}$ band structure at the $\Gamma$ point of the $2 \mathrm{D}$ transverse $\mathrm{BZ}$. The dotted line at $0 \mathrm{eV}$ denotes $E_{\mathrm{F}}$.

$\Delta_{5} \downarrow$ band along the transport direction for energies comprised between -0.8 and $+0.1 \mathrm{eV}$. The minority conductance in this energy range is five orders of magnitude larger $\left(T^{\downarrow} \sim 10^{-7}\right)$ than that for the majority spins. For $E>0.1 \mathrm{eV}$ there is a sharp rise in $T^{\uparrow}$ due to the $\Delta_{1} \uparrow$ band now contributing to the conductance. In the energy window $0.3<E<0.8 \mathrm{eV}$ there are no minority states available and $T^{\downarrow}$ drastically drops. A similar drop due to the lack of minority $\mathrm{SrRuO}_{3}$ bands is found at $-2.5 \mathrm{eV}$. In the AP configuration the electron transmission occurs between majority (minority) states in the left-hand side electrode and minority (majority) in the right-hand side one, so that $T(E)$ for both the spins (identical) is essentially a convolution of those for the majority and minority spin channels of the PA state. As a consequence there is a drastic suppression of $T(E)$ in the regions $-0.8<$ $E<0.1 \mathrm{eV}$ and $0.3<E<0.8 \mathrm{eV}$, where, respectively, the $\Delta_{5} \downarrow$ and $\Delta_{1} \uparrow$ bands in one electrode are not paired in the other. In particular, $T\left(E_{\mathrm{F}}\right)$ for the AP configuration is orders of magnitude smaller than in the PA one. Note that our discussion is based on the band structure at the $\Gamma$ point of the $2 \mathrm{D}$ transverse $\mathrm{BZ}$, for which the decay is the smallest and the transmission the largest. However, also Bloch states with a larger transverse wave vector contribute to the transport and produce a residual transmission. The high transmission below $-1.8 \mathrm{eV}$ corresponds to conduction through the $\mathrm{BaTiO}_{3}$ valence band while that above $0.75 \mathrm{eV}$ corresponds to the conduction band.

\section{I-V curves and bias-dependent TMR}

The spin-polarized current for both the PA and AP configurations and for both the NFE (top panel) and FE (middle panel) structures are shown in Fig. 4, where we focus on the low voltage region in which the current is due entirely to tunneling (the broader $I-V$ are displayed in the insets). The most distinctive feature emerging from the $I-V$ curves is the presence of negative differential resistances (NDR) for the PA

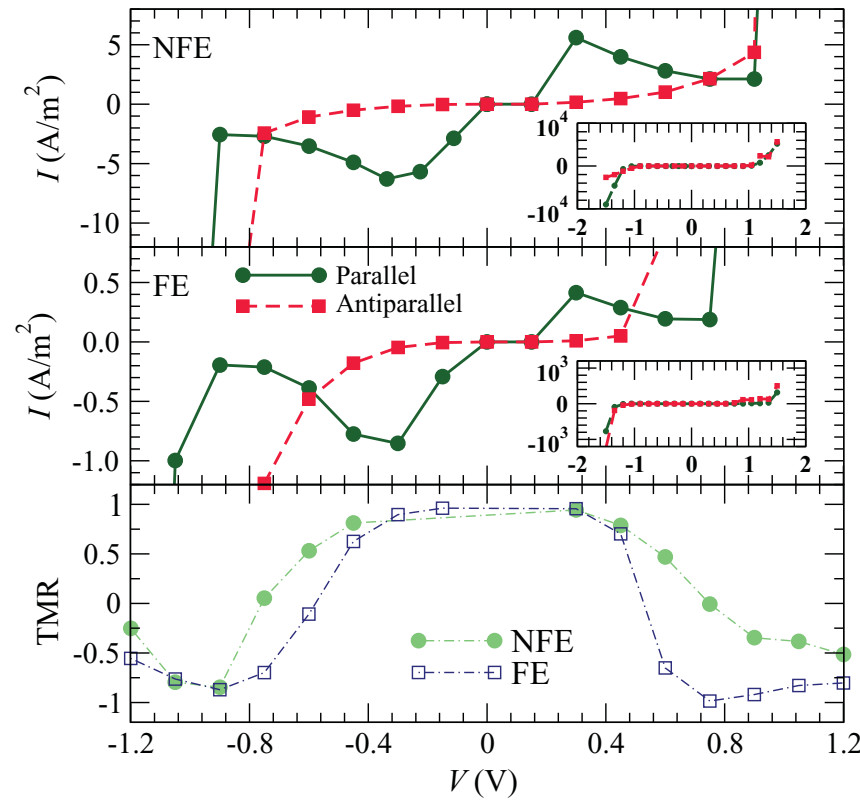

FIG. 4. (Color online) Total current per unit area $I$ as a function of voltage $V$ for the NFE (top panel) and FE (middle panel) structures. In the bottom panel we present the TMR as a function of voltage for both the geometries. In the insets the $I-V$ is presented over a larger current range (the units are the same as in the main figure). Note that at the onset of the $\mathrm{BaTiO}_{3}$ conduction and valence bands the current increases by three orders of magnitude over its low bias value.

alignment, originating from the movement of the $\Delta_{1} \uparrow$ band edge with $V$. Because of the NDR the relative magnitude of the current for the parallel $\left(I^{\mathrm{PA}}\right)$ and antiparallel alignment $\left(I^{\mathrm{AP}}\right)$ can be reversed (i.e., the TMR changes sign with $V$ ). This is demonstrated in the lower panel of Fig. 4, where we present the "pessimistic" TMR ratio, TMR $=\left(I^{\mathrm{PA}}-I^{\mathrm{AP}}\right) /\left(I^{\mathrm{PA}}+I^{\mathrm{AP}}\right)$,
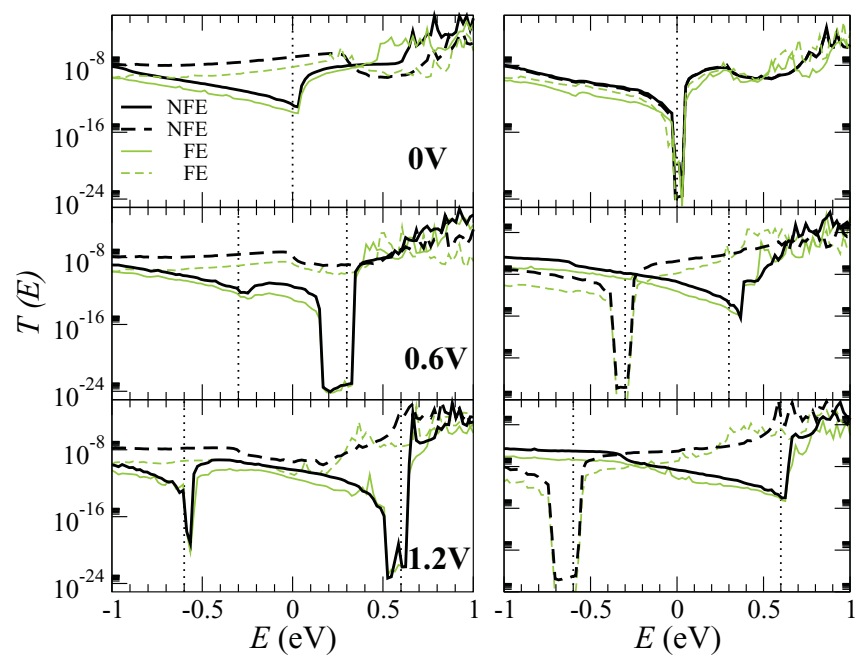

FIG. 5. (Color online) Transmission coefficient $T(E ; V)$ as a function of energy and for different bias voltages. The left panels are for the parallel configuration and the right ones for the antiparallel. In the same figure we report data for both the NFE structure ( $\uparrow$ thick black solid line, $\downarrow$ thick black dashed line) and for the PE one ( $\uparrow$ thin green solid line, $\downarrow$ thin green dashed line). The vertical dotted lines mark the borders of the bias window. 

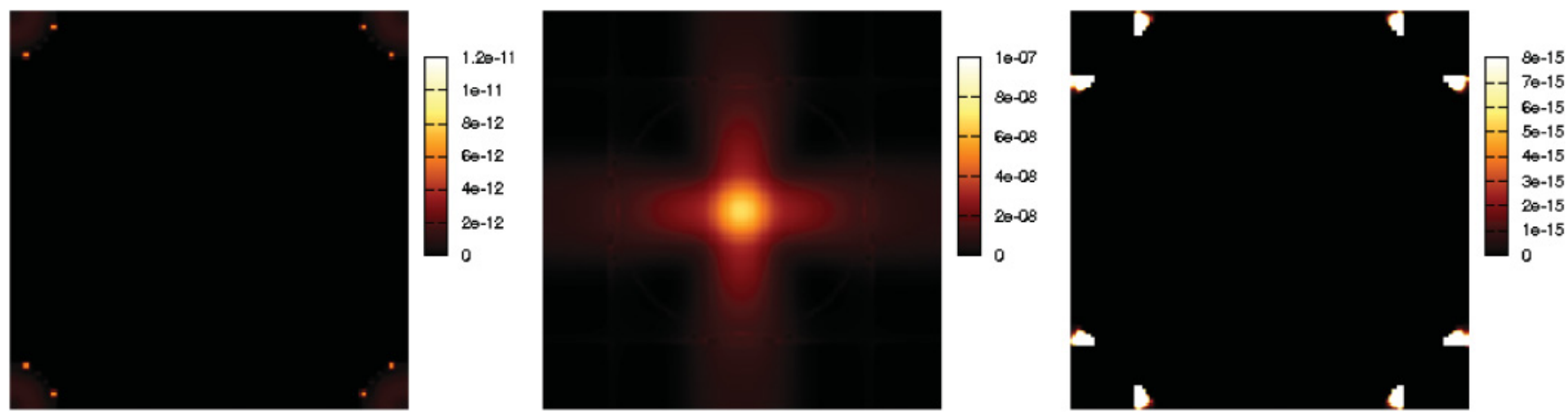

FIG. 6. (Color online) $k$-resolved transmission coefficient $T(E ; V)$ in the 2D Brillioun zone perpendicular to the direction of transport at the Fermi energy. (a) Parallel majority, (b) parallel minority, and (c) antiparallel majority.

as a function of bias. Clearly TMR sign inversion is observed for both the NFE and the FE junctions for voltages in the range $0.7-0.9 \mathrm{~V}$. Furthermore for $V \sim 0.7 \mathrm{~V}$ the TMR for the NFE junction is positive, while that of the FE one is negative, meaning that subtle changes in the barrier electronic structure, such as those induced by ferroelectricity, are sufficient to change the sign of the TMR. Note also that the TMR values reported here are actually extremely large. For instance, for both the NFE and FE junctions and voltages $|V|<0.4 \mathrm{~V}$ the optimistic TMR $\left[\left(I^{\mathrm{PA}}-I^{\mathrm{AP}}\right) / I^{\mathrm{AP}}\right]$ is around $5000 \%$.

The $I-V$ curve can be rationalized by looking at the dependence of $T(E)$ on the bias, ${ }^{25}$ which is presented in Fig. 5. This is mainly determined by the shift of the electrodes' $\Delta_{1}$ and $\Delta_{5}$ band edges with $V$ : for positive voltage the band structure of the left electrode is shifted by $+e V / 2$ ( $e$ is the electron charge) and that of the right one by $-e V / 2$. At a given energy a large $T$ is found only if a band of the same symmetry and spin is found in both electrodes at that energy. For PA alignment at $V=0$, the minority spins dominate the transmission up to $0.3 \mathrm{eV}$, after which one encounters the $\Delta_{5}^{\downarrow}$ upper band edge and $T^{\downarrow}$ is drastically reduced. As $V$ is applied, the $\Delta_{5}^{\downarrow}$ band edge is shifted to lower energies in the right electrode (for $V>0$ ), so that for $V=0.6 \mathrm{~V}$ the high transmission region extends only up to $E_{\mathrm{F}}$, and for $V=1.2 \mathrm{~V}$ it extends only up to $E_{\mathrm{F}}-0.3 \mathrm{eV}$. This is the origin of the NDR found for the PA alignment. In contrast for the AP configuration $T$ is small for energies below $0.1 \mathrm{eV}$ after which it drastically increases because of the $\Delta_{1}$ conduction bands (see Fig. 3). With increasing $V$ the $\Delta_{1}$ band in the right electrode is shifted to lower energies, so that there is a rather large transmission inside the bias window, and eventually the AP current therefore becomes larger than the PA one. This results in the TMR sign change at about $0.7-0.9 \mathrm{~V}$.

\section{Effect of ferrolectric ordering}

The main effect of the ferroelectric order on the transport is an increase of the $\mathrm{BaTiO}_{3}$ band gap (i.e., an increase of the $\Delta_{1}$ and $\Delta_{5}$ decay coefficients, see Fig. 2). In particular, states with
$\Delta_{5}$ symmetry decay significantly faster in the FE MTJ with respect to the NFE one. This results in a global reduction of the transmission although other general features remain rather similar in the two cases. The comparison between $T(E ; V)$ for the FE and NFE junctions is also presented in Fig. 5. Below $E_{\mathrm{F}}$ one may note a substantial reduction of the transmission when going from NFE to FE for both PA and AP alignment as a consequence of the increased $\Delta_{5}$ decay rate.

In Fig. 6 the $k$-resolved transmission coefficient is plotted over the full 2D Brillioun zone perpendicular to the transport direction at the Fermi level. Contributions to the majority transmission is mainly from the band edges. In contrast, transmission through the minority spin is centered around the $\bar{\Gamma}$ point, with the region between $\Gamma$ and $X$ also contributing, reflecting the $d$-state symmetry available at $\mathrm{E}_{F}$. As expected, the highest transmission is associated with the $\bar{\Gamma}$ point while transmission from the Brillioun zone edge is up to four orders of magnitude smaller across a range of energies (not shown). Ferroelectric ordering does not change the symmetry of the tunneling transmission in the $k_{x}-k_{y}$ plane.

\section{CONCLUSION}

In conclusion, we have demonstrated huge TMR in an alloxide ferroelectric MTJ, the sign of which can be inverted as the applied bias increases. Furthermore, the sign inversion occurs at different voltages for different ferroic states of the barrier. Our finite-bias results are explained in terms of the electrodes and the barrier band structures. The possibility to control the TMR by manipulating the ferroic state of the barrier in an MTJ opens a potential avenue for the electrical control of magnetic devices.

\section{ACKNOWLEDGMENTS}

This work is supported by Science Foundation Ireland (Grant No. 07/IN.1/I945), by CRANN and by the EU FP7 ATHENA. Computational resources have been provided by the HEA IITAC project managed by TCHPC and by ICHEC.
*Corresponding authors: caffreyn@tcd.ie, sanvitos@tcd.ie

${ }^{1}$ W. H. Butler, X.-G. Zhang, T. C. Schulthess, and J. M. MacLaren, Phys. Rev. B 63, 054416 (2001).
${ }^{2}$ J. Mathon and A. Umerski, Phys. Rev. B 63, 220403 (2001).

${ }^{3}$ S. Parkin, C. Kaiser, A. Panchula, P. Rice, B. Hughes, M. Samant, and S. Yang, Nat. Mater. 3, 862 (2004). 
${ }^{4}$ S. Yuasa, T. Nagahama, A. Fukushima, Y. Suzuki, and K. Ando, Nat. Mater. 3, 868 (2004).

${ }^{5}$ S. Ikeda, J. Hayakawa, Y. Ashizawa, Y. M. Lee, K. Miura, H. Hasegawa, M. Tsunoda, F. Matsukura, and H. Ohno, Appl. Phys. Lett. 93, 082508 (2008).

${ }^{6}$ M. Julliere, Phys. Lett. A 54, 225 (1975).

${ }^{7}$ T. Nagahama, T. S. Santos, and J. S. Moodera, Phys. Rev. Lett. 99, 016602 (2007).

${ }^{8}$ V. Garcia, M. Bibes, L. Bocher, S. Valencia, F. Kronast,

A. Crassous, X. Moya, S. Enouz-Vedrenne, A. Gloter,

D. Imhoff, C. Deranlot, N. D. Mathur, S. Fusil,

K. Bouzehouane, and A. Barthélémy, Science 327, 1106 (2010).

${ }^{9}$ M. Gajek, M. Bibes, S. Fusil, K. Bouzehouane, J. Fontcuberta, A. Barthelemy, and A. Fert, Nat. Mater. 6, 296 (2007).

${ }^{10}$ J. P. Velev, C.-G. Duan, J. D. Burton, A. Smogunov, M. K. Niranjan, E. Tosatti, S. S. Jaswal, and E. Y. Tsymbal, Nano Lett. 9, 427 (2009).

${ }^{11}$ J. Schwarzkopf and R. Fornari, Prog. Cryst. Growth Charact. Mater. 52, 159 (2006).

${ }^{12}$ J. M. Soler, E. Artacho, J. D. Gale, A. García, J. Junquera, P. Ordejón, and D. Sánchez-Portal, J. Phys. Condens. Matter 14, 2745 (2002).
${ }^{13}$ J. P. Perdew, K. Burke, and M. Ernzerhof, Phys. Rev. Lett. 77, 3865 (1996).

${ }^{14}$ J. Junquera and P. Ghosez, Journal of Computational and Theoretical Nanoscience 5, 2071 (2008).

${ }^{15}$ C. D. Pemmaraju, T. Archer, D. Sánchez-Portal, and S. Sanvito, Phys. Rev. B 75, 045101 (2007).

${ }^{16}$ N. M. Caffrey, T. Archer, and S. Sanvito (unpublished).

${ }^{17}$ J. M. Rondinelli, N. M. Caffrey, S. Sanvito, and N. A. Spaldin, Phys. Rev. B 78, 155107 (2008).

${ }^{18}$ A. Droghetti and S. Sanvito, Appl. Phys. Lett. 94, 252505 (2009).

${ }^{19}$ A. R. Rocha, V. M. Garcia-Suarez, S. Bailey, C. Lambert, J. Ferrer, and S. Sanvito, Phys. Rev. B 73, 085414 (2006).

${ }^{20}$ I. Rungger and S. Sanvito, Phys. Rev. B 78, 035407 (2008).

${ }^{21}$ G. Rijnders, D. H. A. Blank, J. Choi, and C.-B. Eom, Appl. Phys. Lett. 84, 505 (2004).

${ }^{22}$ J. Junquera and P. Ghosez, Nature (London) 422, 506 (2003).

${ }^{23}$ G. Gerra, A. K. Tagantsev, N. Setter, and K. Parlinski, Phys. Rev. Lett. 96, 107603 (2006).

${ }^{24}$ B. Nadgorny, M. S. Osofsky, D. J. Singh, G. T. Woods, J. R. J. Soulen, M. K. Lee, S. D. Bu, and C. B. Eom, Appl. Phys. Lett. 82, 427 (2003).

${ }^{25}$ I. Rungger, O. Mryasov, and S. Sanvito, Phys. Rev. B 79, 094414 (2009). 